\title{
Prediction of promiscuous epitopes in NSP2 of Chikungunya virus: An in-silico approach
}

\author{
Fazal, F. ${ }^{1}$, Anwar, T. ${ }^{1}$, Waheed, $\mathrm{Y}^{2^{*}}$ and Parvaiz, F. ${ }^{1^{*}}$ \\ ${ }^{1}$ Department of Biosciences, COMSATS University Islamabad, Pakistan \\ ${ }^{2}$ Foundation University Medical College, Foundation University Islamabad, Pakistan \\ *Corresponding author e-mails: fahed.pervaiz@comsats.edu.pk (Fahed Parvaiz), \\ yasir_waheed_199@yahoo.com (Yasir Waheed) \\ Received 2 December 2019; received in revised form 3 June 2020; accepted 23 June 2020
}

\begin{abstract}
This study is focused towards developing a global consensus sequence of nonstructural protein 2 (NSP2), a protease of Chikungunya Virus (CHIKV) and predict immunogenic promiscuous T-cell epitopes based on various bioinformatics tools. To date, no epitope data is available for the Chikungunya virus in the IEDB database. In this study, 100 available nucleotide sequences of NSP2-CHIKV belonging to different strains were downloaded from the National Centre for Biotechnology Information (NCBI) database. The nucleotide sequences were subjected to translated sequencing using the EXPASY tool followed by protein alignment using the CLC workbench and a global consensus sequence for the respective protein was developed. IEDB tool was used to predict HLA-I and HLA-II binding promiscuous epitopes from the consensus sequence of NSP2-CHIKV. Thirty-four B-cell based epitopes are predicted and the promiscuous epitope is VVDTTGSTKPDPGD at position 341-354. Twenty-six MHC-I short peptide epitopes are predicted to bind with HLA-A. The promiscuous epitopes predicted to bind with HLA-A*01:01 are VTAIVSSLHY, SLSESATMVY, FSKPLVYY, QPTDHVVGEY at positions $317-326,84-93,535-544$ and 15-24 with percentile ranks $0.17,0.39,0.51$ and 0.81 , respectively. Twenty-four MHC-II short peptide epitopes are predicted for HLA-DRB. The promiscuous epitope predicted to bind with HLA-DRB*01:01 is VVGEYLVLSPQTVLRS from 20-35 with a lowest percentile rank of 0.01 . These predicted epitopes can be effective targets towards development of vaccine against CHIKV. Epitopes predicted in this study displayed good binding affinity, antigenicity and promiscuity for the HLA classes. These predicted epitopes can prove to be translationally important towards the development of CHIKV.
\end{abstract}

\section{INTRODUCTION}

CHIKV is an alphavirus that causes arthralgia and is transmitted through mosquito bites. CHIKV is not fatal yet its symptoms can be severe and disturbing. These symptoms include fever, joint pain, muscle pain, joint swelling, headache, back pain, nausea, maculopapular rash on trunk and extremities of body. CHIKV was first isolated from human serum in Tanzania, 1952. In 2004 there was an outbreak of this disease, which spread through Africa, South East Asia and India with large sporadic epidemics (Waheed et al., 2017). CHIKV is transmitted by Aedes agypti and Aedes albopictus (Petersen \& Powers, 2016). Humans play the role of chief carriers and multipliers of the disease. CHIKV circulates in the blood stream of an infected individual and upon the bite of a naive mosquito (Aedes agypti) on an infected individual, the mosquito will acquire the virus (Rausalu et al., 2016). This infection is likely to take place in elderly individuals or patients with a suppressed immune response.

CHIKV is a positive single stranded RNA virus with a genome size of $12 \mathrm{~kb}$. It encodes two polyproteins, the non-structural polyprotein which consists of four proteins (NSP1, NSP2, NSP3 and NSP4) and the 
structural polyprotein, which consists of five proteins (E3, E2, 6K, E1 and Capsid) (Jain et al., 2017; Solignat et al., 2009; Thiboutot et $a l ., 2010)$. A previous study suggested that NSP2 is involved in the activation of the viral replication complex through its protease and helicase activity (Law et al., 2019). In NSP2, the protease domain lies at the Cterminal domain (471-798) and the helicase domain at the $\mathrm{N}$ terminal (1-470) (Jadav et al., 2012; Jain et al., 2017). Replication and proliferation of CHIKV relies upon protease activity of the NSP2 protein because it cleaves the non-structural polyprotein (NSP1-4) replication complex into individual functional units. Furthermore, NSP2 assumes a significant role in host T-cell shut off, inhibition of antiviral reactions, enhanced virus gene expression and evasion host immune response (Bouraï et al., 2012; Jadav et al., 2012).

CHIKV has infected more than 165,000 individuals in Asia, Africa and the Pacific Islands. Fifty-seven cases of CHIKV reported in US have been associated with traveling. The possibility of CHIKV outbreaks in the US is a real public health threat (Thiberville et al., 2013; Yactayo et al., 2016). In 2016, an outbreak of CHIKV occurred in Karachi, Pakistan. According to different medical sources, approximately 30,000 individuals were infected (Ali \& Dasti, 2018). Since 2004 there have been massive outbreaks of this virus and there is no FDA approved vaccine available for this disease up till now. The possibility of CHIKV outbreaks all over the world is a real public health threat. This outbreak may get complicated with time due to poor sanitation systems, prolonged periods of warm weather, less effective mosquito eradication methods and stagnant water in different areas of the epidemic countries.

This study targets the protease/helicase protein of CHIKV for the prediction of promiscuous epitopes. Different available online tools were used for the future development of an effective and safe epitope-based vaccine against CHIKV.

\section{MATERIALS AND METHODS}

In this study, NSP2-CHIKV, a protease and a helicase, was observed through in-silico tools. To analyze it, a phylogenetic tree and global consensus sequence for NSP2 was developed that was used forprediction of epitopes using various bioinformatics-based tools.

\section{Extraction of Sequences}

One hundred homologous sequences of NSP2-CHIKV from different regions of the world were retrieved from NCBI. The nucleotide sequences were translated to amino acid sequences using EXPASY.

\section{Multiple Sequence Alignment}

All amino acid sequences, along their accession numbers and country names, were fed into CLC main Workbench (version 8.1) using the default setting to develop multiple sequence alignment global consensus sequences and a phylogenetic tree.

\section{Epitope Prediction}

B-cell and T-cell epitopes were predicted from global consensus sequences of NSP2CHIKV using IEDB, an online bioinformatics tool. T-cell based immune epitopes against NSP2-CHIKV for multiple alleles of humans and for various species can be predicted using IEDB.

\section{B-cell Epitope Prediction}

Prediction of B-cell linear epitopes of NSP2CHIKV was performed with BepiPred Linear Epitope Prediction method using IEDB. The threshold was set to 0.35 to get accurate results with good specificity and sensitivity.

\section{T-cell Epitope Prediction}

Peptides binding to MHC-I and MHC-II molecules were predicted using the IEDB tool. Peptides with high antigenicity binding to MHC-I were predicted for HLA-A*01 and peptides binding to MHC-II were predicted for HLA-DRB*01. HLA-A*01 and HLADRB*01 are genes which encode human protein to produce immunity. 


\section{RESULTS}

\section{Protein Alignment}

Multiple protein sequence alignment reveals that NSP2 includes highly conserved residues while certain regions display variations. Conserved regions are shown with dots (Figure 1). Amino acids that are highly variable are denoted by respective amino acid residues. The alignment analysis clearly shows that there is high similarity among sequences of different strains from different countries. The residues having the greatest number of mutations are at positions 54, 374, 642, 643 and 793 as shown in Figure 1.
At position 54, Asparagine ( $\mathrm{N}$ ) is mutated to $\mathrm{S}$ (Serine) in 29 strains from different countries. Interestingly, they are both polar with no charge with a characteristic feature of H-bonding, suggesting no impact on functioning of NSP2.

At position 374, Tyrosine $(\mathrm{Y})$ is mutated to Histidine $(\mathrm{H})$ in 17 strains. Tyrosine is a polar amino acid with no charge while Histidine is a polar amino acid with positive charge, so this represents a shift from neutral to basic amino acid.

At position 642, there is a slight mutation at the Tyrosine residue with Cystine (C ). In 07 strains at position 642 , Tyrosine is mutated

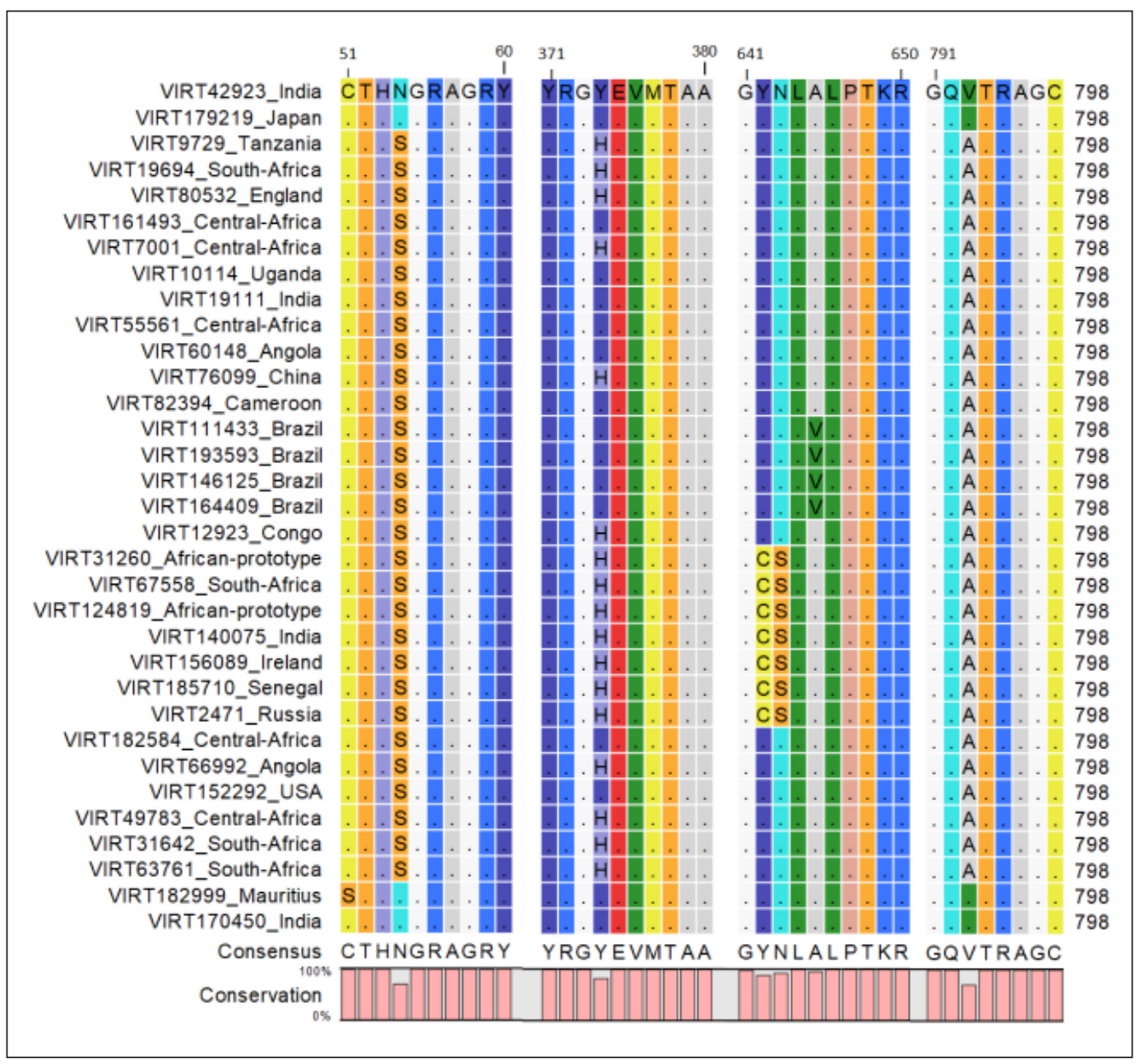

Figure 1. Amino acid sequence alignment of NSP2-CHIKV.

Analysis was performed with CLC main Workbench. Points represent conserved residues while mutated residues were only mentioned with their respective symbols. 
to Cysteine (C). As both residues are polar, it is suggested that there is no impact on the function of NSP2. At position 643, 7 strains have mutations and these mutations are also found in strains from those countries which had the same mutations at position 642. N (Asparagine) is mutated to $\mathrm{S}$ (Serine), both are polar with no charge.

At position 793 , Valine $(\mathrm{V})$ is mutated to Alanine (A) in 29 different strains belonging to different countries. Interestingly, both are non-polar. Most interestingly, these 29 mutations at position 793 are found in strains from the same countries which have mutations at position 54, 374, 642 and 643 . It shows there is some sort of linkage between strains of these different countries which include Tanzania, South Africa, England, Central Africa, Uganda, India, Angola, China, Cameroon, Brazil, Congo, Ireland, Senegal, USA and Russia. At the bottom we can see bars indicating percentage of residue conservation and variation, as shown in Figure 1.

\section{Phylogenetic Tree}

A phylogenetic tree was constructed from 100 protein sequences of NSP2 CHIKV from all over the world using the UPGMA method (Figure 2). The root bifurcates in two interior branches, which share a common ancestor. First the interior branch further bifurcates into two branches. The first branch makes a short cluster or clade alone containing Reunion, India, Singapore and USA while the second sub-branch again divides into two branches. The second interior branch bifurcates into three branches which is further divided. This phylogenetic tree suggests different strains of NSP2 show many similarities, nevertheless, belonging to distinct countries. Contrarily, some strains are distantly related to each other within the same country. For example, strains from India are distantly related to each other and appear on distant clades; these differences might be due to traveling to other countries where CHIKV is epidemic.

\section{Global Consensus Sequence}

In this study, multiple aligned protein sequences were subjected to analysis to develop a global consensus sequence (Figure 3).

\section{B-Cell and T-Cell Epitope Prediction}

Epitopes are part of the antigen, to which B-cell or T-cell antibodies bind to produce immunity against the antigen.

\section{B-Cell Epitopes Prediction}

At least thirty-four short peptides for B-cell epitopes were predicted from global consensus sequences using the BepiPred epitope prediction method, as shown in Table 1. Based on scoring, the epitope VVDTTGSTKPDPGD at position 341-354 is considered to be most effective. The residues above a threshold of 0.35 have a higher probability of being part of the epitope and are represented as yellow, as shown in Figure 4 . The higher the peak in a graph, the higher is the probability of being an effective epitope. If the threshold is set as high as 1, perfect specificity of the epitope for B-cells will be obtained but sensitivity will not be ideal, but at 0.35 default threshold, the correct specificity and sensitivity both will be obtained.

\section{T-Cell Epitope Prediction}

T-cell epitopes encode for cell surface proteins. MHC-I is found in all nucleated cells, which includes B-cells, dendritic cells, antigen presenting cells and macrophages. MHC-I molecules present antigens to cytotoxic T-cells having $\mathrm{CD}^{+}$receptors. On the other hand, MHC-II molecules are usually found on professional antigen presenting cells which include mononuclear phagocytes, dendritic cells, thymic epithelial cells, B cells and some endothelial cells. It represents antigens to helper T-cells having $\mathrm{CD} 4^{+}$receptors. 


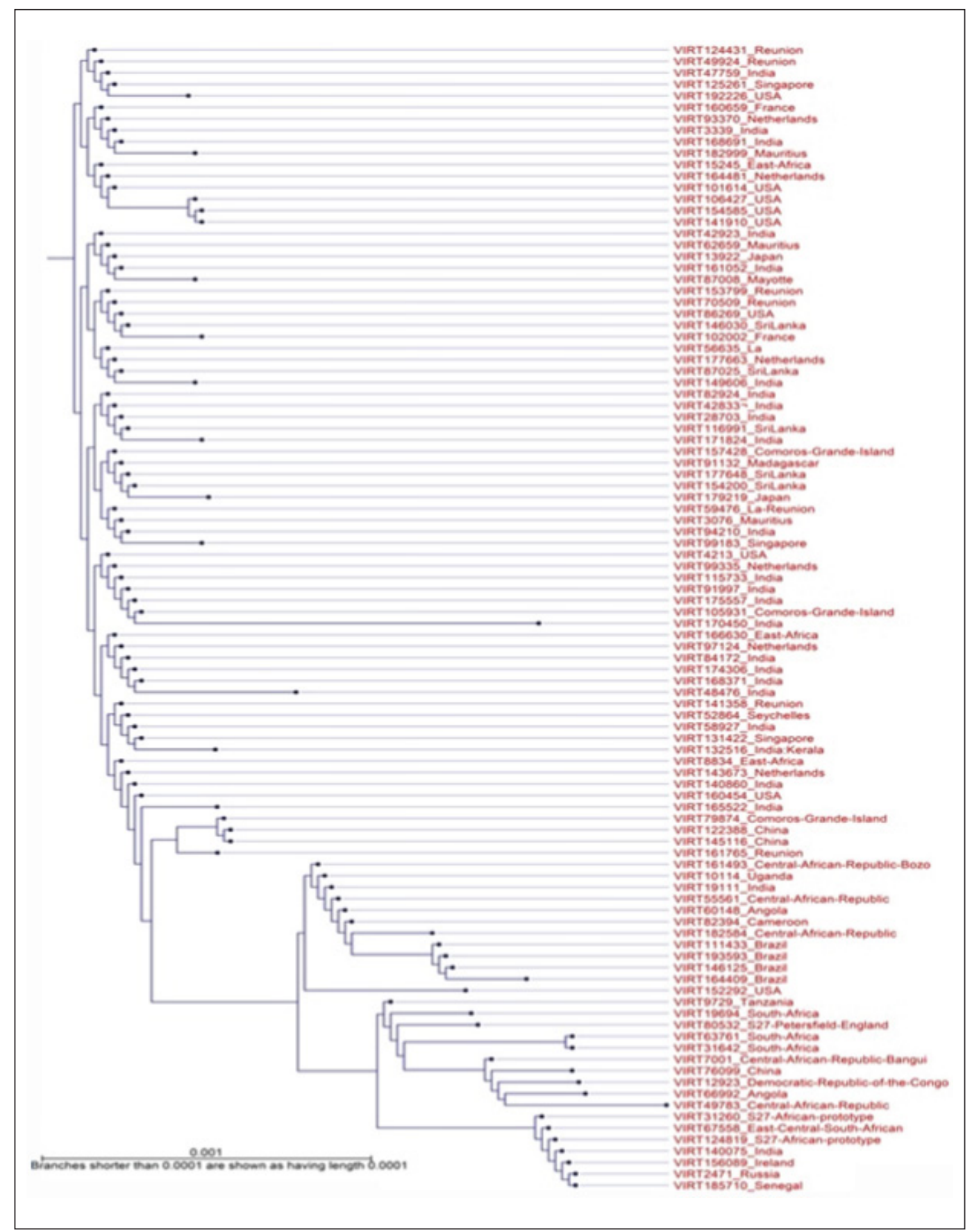

Figure 2. Phylogenetic tree of 100 homologous sequences of NSP2-CHIKV.

The phylogenetic analysis was inferred using the UPGMA method.

\section{MHC-I T-cell Epitopes}

Short peptide epitopes predicted for HLA-A are shown in Table 2. The lower the percentile rank, the higher is the affinity for the epitope. The best epitopes predicted for
HLAA*01:01 are VTAIVSSLHY, SLSESATMVY, FSKPLVYY, QPTDHVVGEY at positions 317326, 84-93, 535-544 and 15-24, respectively. They have the lowest percentile rank. 


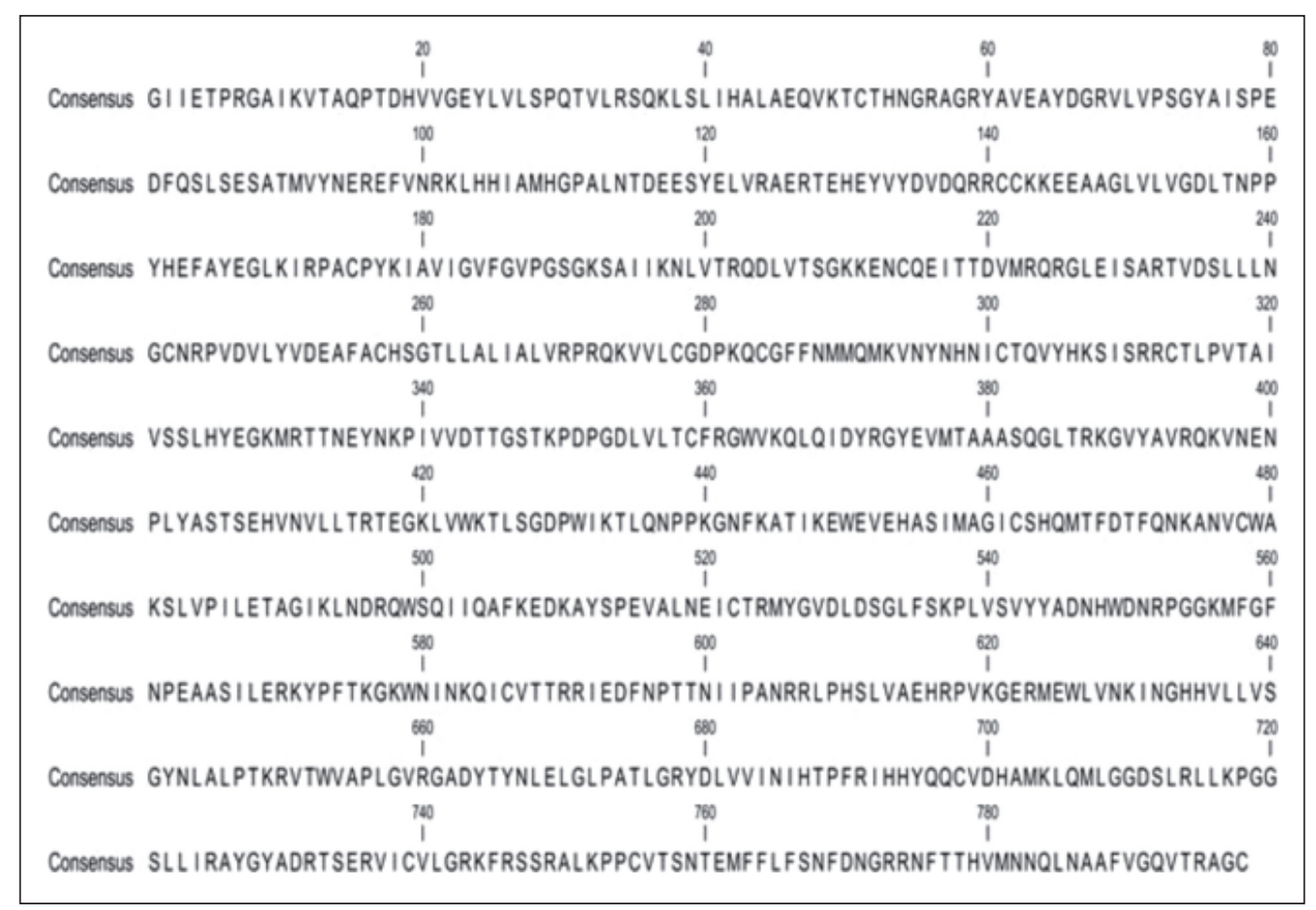

Figure 3. Global Consensus Sequence for NSP2-CHIKV.

The global consensus sequence was achieved for NSP2 of CHIKV using CLC workbench.

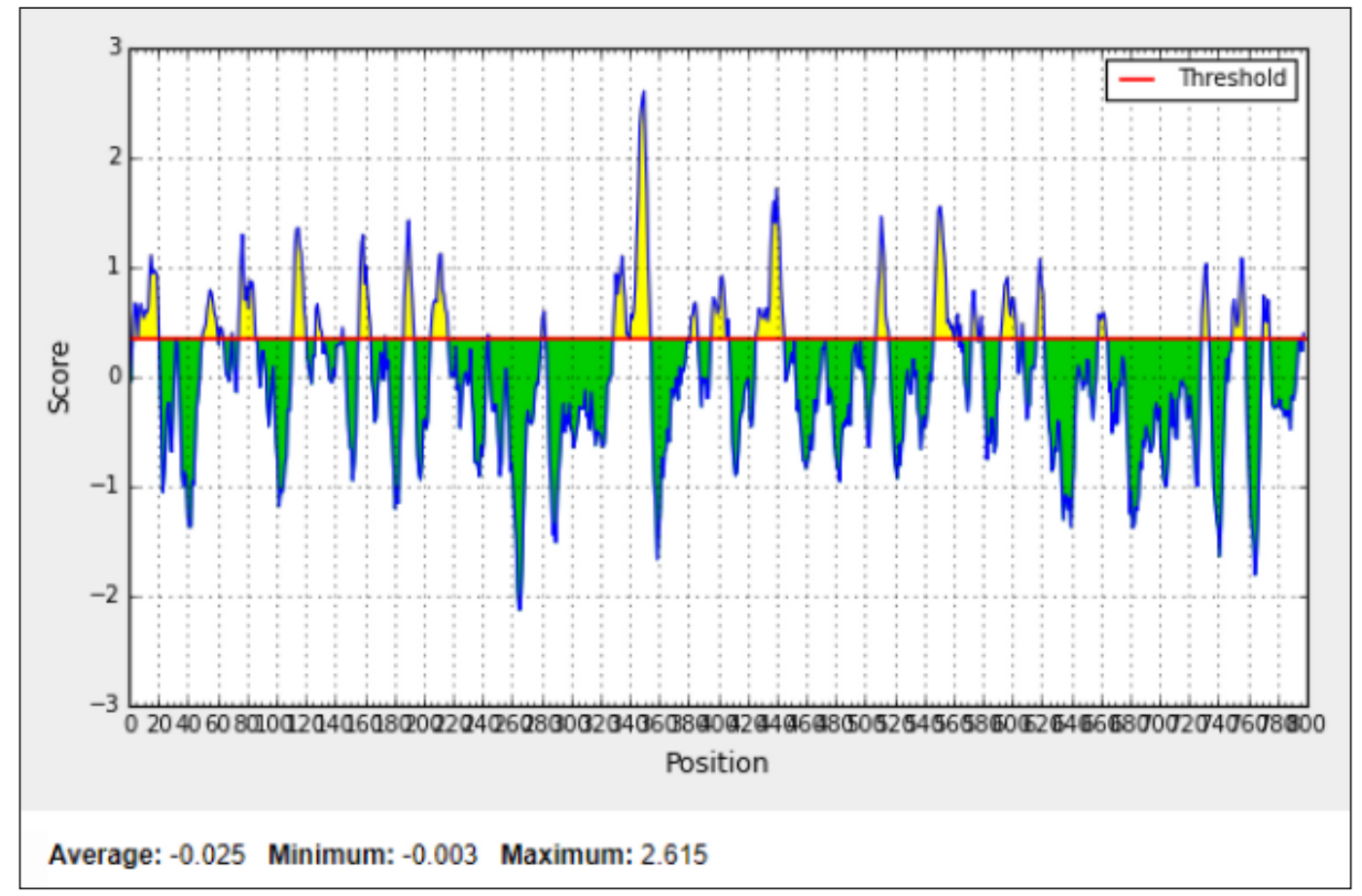

Figure 4. B-cell epitope prediction.

Prediction of B-cell Linear Epitopes of NSP2-CHIKV was done using IEDB. Threshold was set to 0.35 to come up with high specificity and sensitivity. 
Table 1. Prediction of short peptides for B cell epitopes

Predicted peptides:

\begin{tabular}{|c|c|c|c|c|}
\hline No. $\approx$ & Start $\rightleftharpoons$ & End $=$ & Peptide & Length $*$ \\
\hline 1 & 3 & 20 & IETPRGAIKVTAQPTDHV & 18 \\
\hline 2 & 50 & 60 & TCTHNGRAGRY & 11 \\
\hline 3 & 62 & 64 & VEA & 3 \\
\hline 4 & 70 & 70 & L & 1 \\
\hline 5 & 75 & 85 & YAISPEDFQSL & 11 \\
\hline 6 & 111 & 120 & PALNTDEESY & 10 \\
\hline 7 & 127 & 131 & RTEHE & 5 \\
\hline 8 & 145 & 145 & $E$ & 1 \\
\hline 9 & 156 & 164 & LTNPPYHEF & 9 \\
\hline 10 & 174 & 174 & A & 1 \\
\hline 11 & 187 & 193 & VPGSGKS & 7 \\
\hline 12 & 205 & 216 & LVTSGKKENCQE & 12 \\
\hline 13 & 244 & 244 & R & 1 \\
\hline 14 & 281 & 283 & PKQ & 3 \\
\hline 15 & 329 & 339 & KMRTTNEYNKP & 11 \\
\hline 16 & 341 & 354 & VVDTTGSTKPDPGD & 14 \\
\hline 17 & 380 & 380 & A & 1 \\
\hline 18 & 382 & 386 & SQGLT & 5 \\
\hline 19 & 396 & 407 & KVNENPLYASTS & 12 \\
\hline 20 & 426 & 445 & LSGDPWIKTLQNPPKGNFKA & 20 \\
\hline 21 & 497 & 497 & R & 1 \\
\hline 22 & 508 & 516 & EDKAYSPEV & 9 \\
\hline 23 & 547 & 560 & NHWDNRPGGKMFGF & 14 \\
\hline 24 & 562 & 563 & PE & 2 \\
\hline 25 & 565 & 565 & A & 1 \\
\hline 26 & 573 & 576 & PFTK & 4 \\
\hline 27 & 578 & 580 & KWN & 3 \\
\hline 28 & 593 & 603 & EDFNPTTNIIP & 11 \\
\hline 29 & 607 & 607 & $R$ & 1 \\
\hline 30 & 617 & 621 & RPVKG & 5 \\
\hline 31 & 659 & 664 & VRGADY & 6 \\
\hline 32 & 729 & 733 & YADRT & 5 \\
\hline 33 & 750 & 758 & ALKPPCVTS & 9 \\
\hline 34 & 771 & 775 & NGRRN & 5 \\
\hline
\end{tabular}


Table 2. T-cell class I MHC-specific predicted epitopes of the CHIKV polyprotein and their number of alleles and immunogenicity score

\begin{tabular}{|c|c|c|c|c|c|c|}
\hline Allele $\Rightarrow$ & 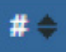 & Start $\approx$ & End $\Rightarrow$ & Length $*$ & Peptide $\Leftrightarrow$ & Consensus Percentile Rank \\
\hline HLA-A*01:01 & 1 & 317 & 326 & 10 & VTAIVSSLHY & 0.175 \\
\hline HLA-A01:01 & 1 & 84 & 93 & 10 & SLSESATMMY & 0.39499999999999996 \\
\hline HLA-A*01:01 & 1 & 535 & 544 & 10 & FSKPLVSWY & 0.515 \\
\hline HLA-Aะ01:01 & 1 & 15 & 24 & 10 & QPTDHVVGEY & 0.81 \\
\hline HLA-A01:01 & 1 & 152 & 161 & 10 & LVGDLTNPPY & 1.04 \\
\hline HLA-A*01:01 & 1 & 51 & 60 & 10 & CTHNGRAGRY & 1.1 \\
\hline HLA-Aะ01:01 & 1 & 85 & 94 & 10 & LSESATMVYN & 1.185 \\
\hline HLA-Aะ01:01 & 1 & 114 & 123 & 10 & NTDEESYELV & 1.21 \\
\hline HLA-Aะ01:01 & 1 & 466 & 475 & 10 & MTFDTFQNKA & 1.415 \\
\hline HLA-A*01:01 & 1 & 16 & 25 & 10 & PTDHVVGEYL & 1.435 \\
\hline HLA-A*01:01 & 1 & 123 & 132 & 10 & VRAERTEHEY & 1.6 \\
\hline HLA-A*01:01 & 1 & 534 & 543 & 10 & LFSKPLVSVY & 1.9 \\
\hline HLA-A*01:01 & 1 & 757 & 766 & 10 & TSNTEMFFLF & 2.0999999999999996 \\
\hline HLA-A*01:01 & 1 & 720 & 729 & 10 & GSLLIRAYGY & 2.2 \\
\hline HLA-Aะ01:01 & 1 & 406 & 415 & 10 & TSEHVNVLLT & 2.6 \\
\hline HLA-A $01: 01$ & 1 & 517 & 526 & 10 & ALNEICTRMY & 2.65 \\
\hline HLA-Aะ01:01 & 1 & 686 & 695 & 10 & IHTPFRIHHY & 2.6500000000000004 \\
\hline HLA-Aะ01:01 & 1 & 156 & 165 & 10 & LTNPPYHEFA & 2.7 \\
\hline HLA-A*01:01 & 1 & 563 & 572 & 10 & EAASILERKY & 2.9 \\
\hline HLA-Aะ01:01 & 1 & 127 & 136 & 10 & RTEHEYVYDV & 3.3 \\
\hline HLA-A*01:01 & 1 & 377 & 386 & 10 & MTAAASQGLT & 3.8000000000000003 \\
\hline HLA-Aะ01:01 & 1 & 670 & 679 & 10 & LGLPATLGRY & 3.9 \\
\hline HLA-Aะ01:01 & 1 & 218 & 227 & 10 & TTDVMRQRGL & 3.95 \\
\hline HLA-A 01:01 & 1 & 756 & 765 & 10 & VTSNTEMFFL & 4.199999999999999 \\
\hline HLA-Aะ01:01 & 1 & 755 & 764 & 10 & CVTSNTEMFF & 4.35 \\
\hline HLA-Aะ01:01 & 1 & 89 & 98 & 10 & ATMVYNEREF & 4.5 \\
\hline
\end{tabular}

\section{MHC-II T-cell Epitopes}

Short peptide epitopes predicted for HLA-A are shown in Table 3. The best epitope predicted for HLA-DRB*01:01 is
VVGEYLVLSPQTVLRS from 20-35 with the lowest percentile rank. The lower the percentile rank, the higher is the affinity for the epitope. 
Table 3. T-cell class II MHC-specific predicted epitopes of the CHIKV polyprotein and their number of alleles and immunogenicity score

\begin{tabular}{|c|c|c|c|c|c|}
\hline Allele & $\#$ \# & 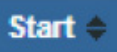 & End $*$ & Peptide & Consensus percentile rank $\mathbf{}$ \\
\hline HLA-DRB1*01:01 & 1 & 20 & 34 & WVGEYLVLSPQTVLR & 0.01 \\
\hline HLA-DRB1*01:01 & 1 & 21 & 35 & VGEYLVLSPQTVLRS & 0.01 \\
\hline HLA-DRB1*01:01 & 1 & 22 & 36 & GEYLVLSPQTVLRSQ & 0.03 \\
\hline HLA-DRB1*01:01 & 1 & 19 & 33 & HVVGEYLVLSPQTVL & 0.05 \\
\hline HLA-DRB1*01:01 & 1 & 23 & 37 & EYLVLSPQTVLRSQK & 0.09 \\
\hline HLA-DRB1*01:01 & 1 & 371 & 385 & YRGYEVMTAAASQGL & 0.09 \\
\hline HLA-DRB1×01:01 & 1 & 24 & 38 & YLVLSPQTVLRSQKL & 0.19 \\
\hline HLA-DRB1*01:01 & 1 & 18 & 32 & DHWGEYLVLSPQTV & 0.28 \\
\hline HLA-DRB1*01:01 & 1 & 372 & 386 & RGYEVMTAAASQGLT & 0.32 \\
\hline HLA-DRB1*01:01 & 1 & 370 & 384 & DYRGYEVMTAAASQG & 0.47 \\
\hline HLA-DRB1*01:01 & 1 & 369 & 383 & IDYRGYEVMTAAASQ & 0.79 \\
\hline HLA-DRB1*01:01 & 1 & 373 & 387 & GYEVMTAAASQGLTR & 0.88 \\
\hline HLA-DRB1*01:01 & 1 & 368 & 382 & QIDYRGYEVMTAAAS & 0.96 \\
\hline HLA-DRB1*01:01 & 1 & 374 & 388 & YEVMTTAAASQGLTRK & 1.71 \\
\hline HLA-DRB1*01:01 & 1 & 117 & 131 & EESYELVRAERTEHE & 2.27 \\
\hline HLA-DRB1*01:01 & 1 & 710 & 724 & GDSLRLLKPGGSLLI & 2.51 \\
\hline HLA-DRB1*01:01 & 1 & 711 & 725 & DSLRLLKPGGSLLIR & 2.51 \\
\hline HLA-DRB1*01:01 & 1 & 116 & 130 & DEESYELVRAERTEH & 2.73 \\
\hline HLA-DRB1*01:01 & 1 & 256 & 270 & ACHSGTLLALIALVR & 3.24 \\
\hline HLA-DRB1*01:01 & 1 & 257 & 271 & CHSGTLLALIALVRP & 3.49 \\
\hline HLA-DRB1*01:01 & 1 & 258 & 272 & HSGTLLALIALVRPR & 3.49 \\
\hline HLA-DRB1×01:01 & 1 & 259 & 273 & SGTLLALIALVRPRQ & 3.49 \\
\hline HLA-DRB1×01:01 & 1 & 118 & 132 & ESYELVRAERTEHEY & 3.63 \\
\hline HLA-DRB1*01:01 & 1 & 260 & 274 & GTLLALIALVRPRQK & 3.72 \\
\hline
\end{tabular}

\section{DISCUSSION}

CHIKV is likely to be transmitted through Aedes agypti and Aedes albopictus (Petersen \& Powers, 2016). This infection takes place in aged individuals or patients with a compromised immune response. Currently, there is no FDA-approved vaccine against
CHIKV (Law et al., 2019). Furthermore, there is no specific treatment against this disease and the generalized medication involves use of paracetamol, aspirin and anti-inflammatory drugs (Thiberville $e t$ al., 2013). It is estimated that CHIKV evolved from ancestors that existed 500 years ago and the lineage is thought to start within 
Africa. It is estimated that 70 to 90 years ago CHIKV was introduced into Asia from Africa (Volk et al., 2010).

Previous studies demonstrated structural and non-structural proteins as targets to find the antigenic epitopes that could be effectively used as vaccine candidates (Islam et al., 2012; Kori et al., 2015; Nair et al., 2015; Rashad et al., 2013). Epitope prediction in a few studies is based on a single gene (NSP4, RNA-dependent RNA Polymerase) of CHIKV (Waheed et al., 2017) or interaction of two CHIKV genes (NSP1 interaction with NSP2) (Kumar et al., 2018). NSP2 and NSP3 of CHIKV exhibit activity of RNA interface (RNAi) suppression. RNAi is an antiviral mechanism pathway (Mathur et al., 2016). The ability to recognise small peptide proteins of NSP2 CHIKV differs from NSP2 of other Alphaviruses (Saisawang et al., 2015).

Humoral immunity is induced by B-cell epitope interaction with B-lymphocytes, followed by production of antibody-secreting plasma cells and memory cells (Shawan et al., 2014). Vaccines have mostly been based on B-cell immunity (Parashar \& Cherian, 2014; Powers, 2018) but in-silico approaches made it possible to induce T-cell immunity by predicting and designing vaccines against T-cell epitopes, which is supported because $\mathrm{CD}^{+}$cells generate strong immunity against infected cells (Yasmin \& Nabi, 2016). Epitope vaccines are easy to develop; manufacturing is cheap and has fewer side effects compared to conventional epitopes (Dar et al., 2016). Epitope-based vaccines are thought to be promising vaccines for the next generation (Ayub, G et al.). MHC is a tightly linked set of genes that play an essential role in adaptive immunity and define the outcome of many host immune responses. Furthermore, the immunogenicity of the potential epitope is defined by its binding affinity to MHC molecule (Kumar Pandey et al., 2018).

In the present study, NSP2-CHIKV has been analyzed using various in-silico tools. One hundred homologous sequences have been retrieved from databases and analyzed with the aim of determining the best possible epitopes with high binding affinity and antigenicity.

We designed short peptides of amino acids/epitopes from highly conserved regions of NSP2-CHIKV. It is suggested that these epitopes could be incorporated into vaccine formulations to yield a better outcome in the human population.

This study predicts 34 B-cell epitopes and the promiscuous epitope is VVDTTGSTKPDPGD at position 341-354. Furthermore, 26 MHC-I epitopes are predicted to bind with HLA-A. The promiscuous epitopes include VTAIVSSLHY, SLSESATMVY, FSKPLVYY, and QPTDHVVGEY at positions 317-326, 84-93, 535-544 and $15-24$ with the percentile ranks of $0.17,0.39$, 0.51 and 0.81 , respectively. These have been predicted to bind with HLA-A*01:01. In addition, this study predicts 24 MHC-II short peptide epitopes for HLA-DRB. The promiscuous epitope predicted to bind with HLA-DRB*01:01 is VVGEYLVLSPQTVLRS from 20-35 with lowest percentile rank of 0.01 . These epitopes are suggested to be incorporated in the vaccine formulations to yield better status of human population.

CHIKV is evolving with time due to different reasons such as traveling, urbanization, lack of effective control measures, adaptation of virus and spread of new vectors and there should be an effective means to stop this disease from spreading. This study was conducted to analyze NSP2-CHIKV as it plays a crucial role in CHIKV replication so that epitopes can be analyzed for B-cell and T-cell affinity as effective antiviral drug design.

\section{Conflict of Interest}

The authors declare that there is no conflict of interest.

\section{REFERENCES}

Ali, I. \& Dasti, J.I. (2018). Chikungunya virus: an emerging arbovirus in Pakistan. Journal of Pakistan Medical Association 68: 252-257. 
Bouraï, M. (2012). Mapping of Chikungunya virus interactions with host proteins identified nsP2 as a highly connected viral component. Journal of Virology $\mathbf{8 6}$ : 3121-3134.

Dar, H. (2016). Prediction of promiscuous T-cell epitopes in the Zika virus polyprotein: An in silico approach. Asian Pacific Journal of Tropical Medicine 9: 844-850.

Islam, R., Sakib, M.S. \& Zaman, A. (2012). A computational assay to design an epitope-based peptide vaccine against chikungunya virus. Future Virology 7: 1029-1042.

Jadav, S.S., Jayaprakash, V., Basu, A. \& Sinha, B.N. (2012). Chikungunya protease domain-high throughput virtual screening. International Scholarly and Scientific Research Innovation 6: 718.

Jain, J. (2017). In silico analysis of natural compounds targeting structural and nonstructural proteins of chikungunya virus. F1000 Research 6: 1601.

Kori, P., Sajjan, S.S. \& Madagi, S.B. (2015). In silico prediction of epitopes for Chikungunya viral strains. Journal of Pharmaceutical Investigation 45: 579591.

Pandey, K.R., Ojha, R., Mishra, A. \& Kumar Prajapati, V. (2018). Designing Band Tcell multiepitope based subunit vaccine using immunoinformatics approach to control Zika virus infection. Journal of Cellular Biochemistry 119: 7631-7642.

Law, Y.S. (2019). Structural insights into RNA recognition by the Chikungunya virus nsP2 helicase. Proceedings of the National Academy of Sciences 116: 9558-9567.

Mathur, K., Anand, A., Dubey, S.K., SananMishra, N., Bhatnagar, R.K. \& Sunil, S. (2016). Analysis of chikungunya virus proteins reveals that non-structural proteins nsP2 and nsP3 exhibit RNA interference (RNAi) suppressor activity. Scientific Reports 6: 38065.
Nair, A.S., Dhar, P.K. \& Nayarisseri, A. (2015). Epitope characterization and docking studies on Chikungunya viral Envelope 2 protein. International Journal of Scientific and Research Publications 5: $1-9$.

Parashar, D. \& Cherian, S. (2014). Antiviral perspectives for chikungunya virus. BioMed Research International 2014 : 1-11.

Petersen, L.R. \& Powers, A.M. (2016). Chikungunya: Epidemiology. F1000 Research 5: 82.

Powers, A.M. (2018). Vaccine and therapeutic options to control chikungunya virus. Clinical Microbiology Reviews 31: e00104-00116.

Rashad, A.A., Mahalingam, S. \& Keller, P.A. (2013). Chikungunya virus: emerging targets and new opportunities for medicinal chemistry. Journal of Medicinal Chemistry 57: 1147-1166.

Rausalu, K. (2016). Chikungunya virus infectivity, RNA replication and nonstructural polyprotein processing depend on the nsP2 protease's active site cysteine residue. Scientific Reports 6: 37124 .

Saisawang, C., Sillapee, P., Sinsirimongkol, K., Ubol, S., Smith, D.R. \& Ketterman, A.J. (2015). Full length and protease domain activity of chikungunya virus nsP2 differ from other alphavirus nsP2 proteases in recognition of small peptide substrates. Bioscience Reports 35: e00196.

Shawan, M.M.A., AlMahmud, H., Hasan, M.M., Parvin, A., Rahman, M.N. \& Rahman, S.B. (2014). In silico modeling and immunoinformatics probing disclose the epitope based peptidevaccine against Zika virus envelope glycoprotein. Indian Journal of Pharmaceutical and Biological Research 2: 44.

Solignat, M., Gay, B., Higgs, S., Briant, L. \& Devaux, C. (2009). Replication cycle of chikungunya: a re-emerging arbovirus. Virology 393: 183-197. 
Thiberville, S.D., Moyen, N., DupuisMaguiraga, L., Nougairede, A., Gould, E.A., Roques, P. \& de Lamballerie, X. (2013). Chikungunya fever: epidemiology, clinical syndrome, pathogenesis and therapy. Antiviral Research 99: 345-370.

Thiboutot, M. (2010). Chikungunya: a potentially emerging epidemic? PLoS Neglected Tropical Diseases 4: e623.

Volk, S.M. (2010). Genome-scale phylogenetic analyses of chikungunya virus reveal independent emergences of recent epidemics and various evolutionary rates. Jourmal of Virology 84: 6497-6504.
Waheed, Y. (2017). Prediction of promiscuous T-cell epitopes in RNA dependent RNA polymerase of Chikungunya virus. Asian Pacific Journal of Tropical Medicine 10: $760-764$.

Yactayo, S. (2016). Epidemiology of Chikungunya in the Americas. The Journal of Infectious Diseases 214: S441-S445.

Yasmin, T. \& Nabi, A.N. (2016). B and T-cell epitopebased peptides predicted from evolutionarily conserved and whole protein sequences of Ebola virus as vaccine targets. Scandinavian Journal of Immunology 83: 321-337. 